LA-UR- $\quad 92-693$

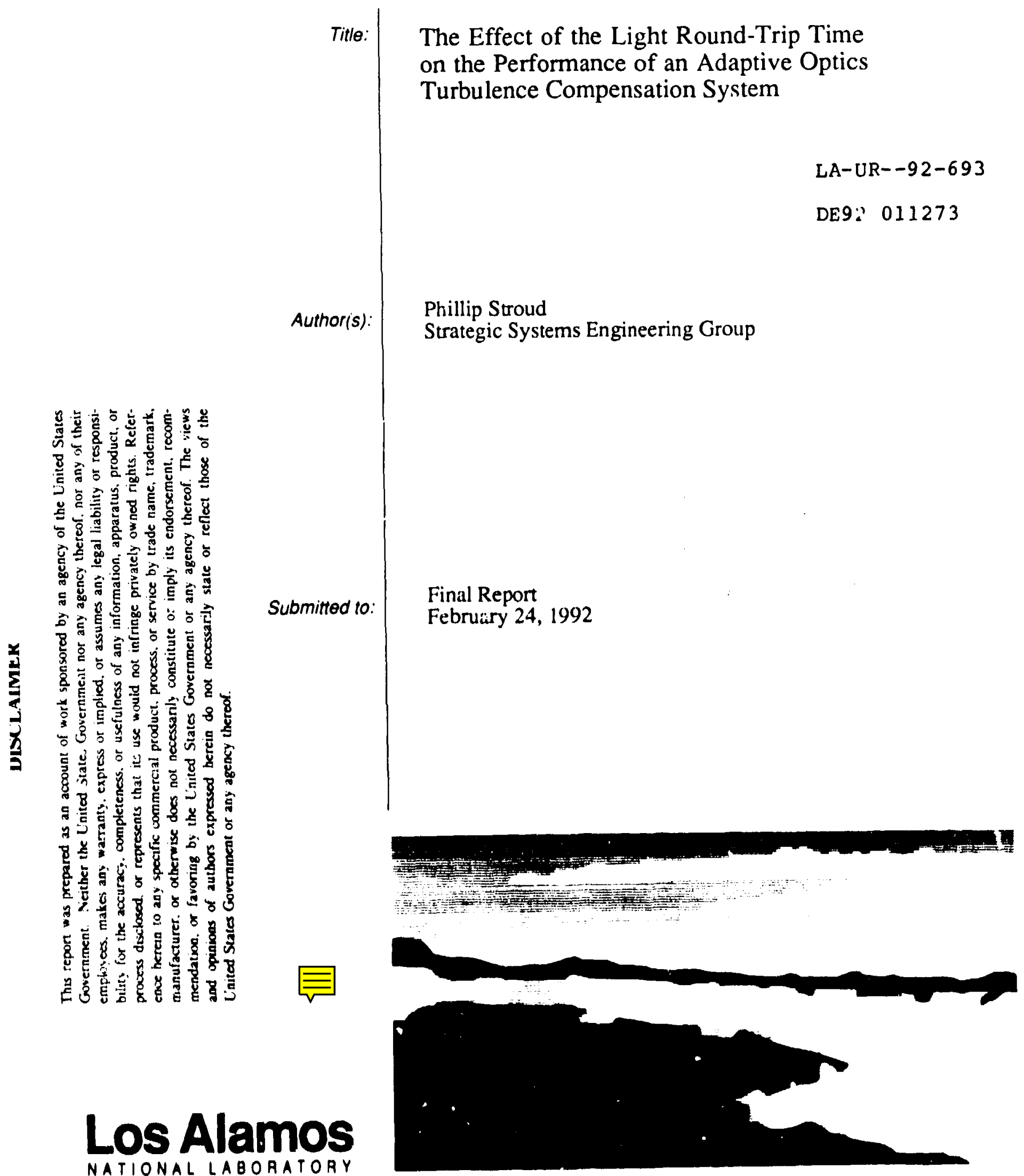

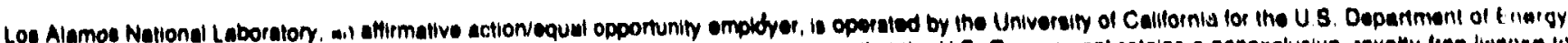

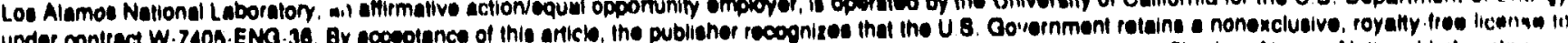

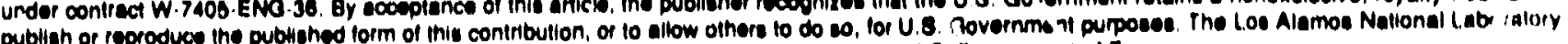
publish or reproduce the published form of this contribution, of to allow others to do 10 , for U.8. Rovernme 71 purposes.

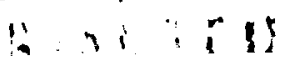

6. ram No nin in 


\title{
The Effect of the Light Round-Trip Time on the Performance of an Adaptive Optics Turbulence Compensation System
}

\author{
Phillip Stroud \\ Los Alamos National Laboratory, Strategic Systems Engineering Group \\ February 24, 1992
}

1. Introduction. The propagation of a laser beam through atmospheric turbulence can, under many circumstances, be compensated to near diffraction limited levels, by use of an adaptive optics system. In the ideal case, a beacon light source is generated at the aimpoint. This light traverses through the turbulent path back to the aperture. The distribution of phase perturbations in the aperture plane is sensed, and the reverse of this phase is applied to the outgoing beam. When the urbulent path is long (e.g. a few hundred kilometers), and the effective wind speed is high (such as is the case when the laser is based on a plane, with airspeeds around $200 \mathrm{~m} / \mathrm{s}$ ), the performance of the adaptive optics system can be significantly degraded due to the time delay from when the beacon samples the turbulence, until the beam propagates through the turbulence. Tr is effect has not been treated in the past, because for astronomical applications, the time delays due to the round trip time of light are too short (tens of micrcieconds) to ciluse problems.

This paper presents an analysis of the anisoplanatic effect of the round irip time of light. A formulation of the phase variance has been constructed for tr:, effert. The tine response of the adaptive optics system (i.e. servo bandwidth) is intimately related to the light round trip time effect, and is included in the analysis. Results are first ohtained for the large aperture limit, and then the effects of finite aperture size are treated. A set of scilling relationships have been found that enable the formulation to be cast in a form that is insensitive to the turbulence strength profile along the propagation path.

2. Whase Varinnce Eormulation. A formulation for the Strehl ratio fior : focused compensated benm propagating through turbulence, was developed in a parent paper |LAUR 92-479|. That formulation can be applied to the anisoplanatic effect ciansed 
by the finite speed of light. In the case that amplitude perturbations are negligible and the Strehls are greater than about 0.3 (the Marechal limit), the Strehl was given by

$$
\delta \equiv \frac{\leq I \geq}{I_{D C}}=\exp \left(-\int_{A}\left\langle\phi_{A}^{2}\right\rangle+\left\langle\int_{A} \int_{B} \phi_{A} \phi_{B}>\right) \equiv \exp \left(-\sigma_{\phi}^{2}\right)\right.
$$

$\phi_{\mathrm{A}}$ is the phase error at position $\vec{\rho}_{\mathrm{A}}$ in the aperture plane. It is the phase perturbation due to turbulence for light propagating from $\vec{\rho}_{A}$ to the aimpoint, minus the phase correction applied at $\vec{\rho}_{A}$. The average over the aperture is designated by $\int_{A}$. The phase variance is the negative of the argument of the exponential, i.e. $\sigma_{\phi}{ }^{2}$.

For a perfect sensor and deformable mirror, using a beacon source located at the aimpoint, the applied phase correction will be the phase increment sensed in the beacon light arriving at $\vec{p}_{\mathrm{A}}$. The effect that will be treated here is the delay between the time the beacon samples a given turbule and the time the uutgoing beam propagates through the turbule. During this delay, the turbule will move due to an effective perpendicular wind, $w(z)$. The outgoing beam will thus propagate through a different turbulence field than the beacon light. This delay will have two components. There is the round trip time of light, given by twice the distance from the aperture to the turbule divided by the speed of light. There will also be a delay due to the finite time between receipt of the beacon signal and emission of the compensated beam. This second delay represents the combined effects of a finite beacon sensor frame tine, a finite processing time, and/or a finite bandwidth $A O$ control loop for the deformable mirror.

In the notation of the previous paper, the phase perturbation received at time 0 , at apertur: position $\vec{\rho}_{A}$, is given by

$\phi_{A} I^{I N}(0)=k_{0} \int_{0}^{L} d z \sqrt{0.066 C_{n}^{2}\left(z_{0}\right)} \int \frac{d k}{k^{11 / 6}}\left[\cos \left(\vec{k}_{\perp} \cdot \vec{\rho}_{\Lambda} t / L+k_{\|}\left(z-z_{0}\right) \cdot \vec{k}_{\perp} \cdot \vec{w} z / c+\varphi\left(\vec{k}, z_{0}\right)\right)\right]$

while the phase perturbation on the outgoing light, emitted at time $\tau$, is given by

$$
\begin{aligned}
& \phi_{A}^{O U T}(\tau)=k_{0} \int_{0}^{L} d z \sqrt{0.066 C_{n}^{2}\left(z_{0}\right)} \frac{d \bar{k}}{k^{11 / 6}} \\
& {\left[\cos \left(\vec{k}_{\perp} \cdot \vec{p}_{A} t / L+k, \|\left(z_{-}-z_{0}\right)+\vec{k}_{\perp} \cdot \vec{w} z / c+\vec{k}_{\perp} \cdot \vec{w} \tau+\varphi\left(k_{,}, z_{(0)}\right)\right)\right]}
\end{aligned}
$$

$k_{0}$ is the laser wavenumber in vacuum, $2 \pi \lambda . z$ is the distance along the propagation path, where, $z=0$ at the apereure, and $z=L$ at the aimpoint. $z_{1}$ ) is an arbitrary point in the vicinity of 
z. $C_{n}^{2}$ is the index of refraction structure coefficient, which charactcrizes the strength of the turbulence. By definition, the expected value of the square of the difference of index of refraction at two points separated by a distance $\vec{r}$ is $\left\langle(n(\vec{r}+\vec{r})-n(\vec{r}))^{2}\right\rangle=C_{n}^{2} r^{2 / 3} . \vec{k}$ is the wavenumber of Fourier cosine expansion component of the turbulence. $t$ is $L-z . \vec{w}$ is the effective wind speed as a function of $z$, with which the turbulence is blowing across the path. $\varphi\left(\bar{k}, z_{0}\right)$ is the phase of the turbulence component, which is a unitormly distributed random variable.

The compensated phase error, obtained by subtracting the phase perturbation received at time 0 from the phase perturbation of the outgoing beam emitted at time $\tau$, is then

$$
\begin{aligned}
\phi_{A}(\tau)=k_{0} \int_{0}^{L} d z \sqrt{ } & 0.066 C_{n}^{2}\left(z_{0}\right) \int \frac{d k}{k^{11 / 6}} \\
& \left\{\left[\cos \left(\vec{k}_{\perp} \cdot \vec{\rho}_{A} L / L+k_{\|}\left(z-z_{0}\right)-\vec{k}_{\perp} \cdot \overrightarrow{k^{\prime}} z / c+\varphi\left(\vec{k}, z_{0}\right)\right)\right]\right. \\
& \left.-\left[\cos \left(\vec{k}_{\perp} \cdot \vec{\rho}_{A} t / L+k_{\|}\left(z-z_{0}\right)+\vec{k}_{\perp} \cdot \vec{w} z / c+\vec{k}_{\perp} \cdot \vec{w} \tau+\varphi\left(\vec{k}, z_{0}\right)\right)\right]\right\}
\end{aligned}
$$

Following the development presented in the previous paper, the phase variance is found to be

$$
\begin{array}{r}
\sigma_{\phi}^{2}=1.303 \mathrm{k}_{0}^{2} \int_{0}^{\mathrm{L}} \mathrm{dz} \mathrm{C}_{\mathrm{n}}^{2}(\mathrm{z}) \int \frac{\mathrm{d} \mathrm{k}_{\perp}}{\mathrm{k}_{\perp}^{8 / 3}}\left\{1-\left[\frac{2 \mathrm{~J}_{1}\left(\mathrm{k}_{\perp} \mathrm{R} \mathrm{R} / \mathrm{L}\right)}{\left(\mathrm{k}_{\perp} \mathrm{R} / \mathrm{L}\right)}\right]^{2}\right\} \\
{\left[2-\mathrm{J}_{0}\left(\mathrm{k}_{\perp} w(\tau-2 z / c)\right)-\mathrm{J}_{0}\left(\mathrm{k}_{\perp} w(\tau+2 \mathrm{z} / \mathrm{c})\right)\right]}
\end{array}
$$

In the limit of large aperture, the $\mathrm{J}_{1}$ ierm of $\mathrm{Eq}[5]$ goes to zero. The phase variance is then

$$
\begin{aligned}
\lim _{R \rightarrow \infty} \sigma_{\phi}^{2} & =1.303 k_{0}^{2} \int_{d}^{L} d z C_{n}^{2}(z) \int \frac{d k_{1}}{k_{\perp}} \frac{8 / 3}{8 / 3}\left[2 \cdot J_{0}\left(k_{\perp} w(\tau-2 z / c)\right)-J_{0}\left(k_{\perp} w(\tau+2 z / c)\right)\right] \\
& =1.457 k_{0}{ }^{2} \int^{L} d z C_{n}^{2}(z) w^{5 / 3} \tau^{5 / 3}\left[\left|1-\frac{2 z}{\tau c}\right|^{5 / 3}-\left(1+\frac{2 z}{\tau c}\right)^{5 / 3}\right]
\end{aligned}
$$


3. Large Aperture. Infinite Light_Speed. Finite_Bandwidth_Case. In the limiting case where the speed of light goes to infinity, the phase variance is

$$
\begin{aligned}
\lim _{\mathrm{R} ; \mathrm{c} \rightarrow \infty} \sigma_{\phi}^{2} & =(20.13 \tau)^{5 / 3}\left[0.01956 \mathrm{k}_{\mathrm{o}}^{2} \int_{0}^{\mathrm{L}} \mathrm{d} z \mathrm{C}_{\mathrm{n}}^{2}(\mathrm{z}) \mathrm{w}^{5 / 3}\right] \\
& =(20.13 \tau)^{5 / 3} \mathrm{f}_{0}^{5 / 3}
\end{aligned}
$$

where $f_{0}$ is Greenwood's frequency, given by

$$
f_{0}=\left[0.01956 \mathrm{k}_{0}{ }^{2} \int_{0}^{L} \mathrm{~d} z C_{n}^{2}(z) w^{5 / 3}\right]^{3 / s}
$$

The delay time, $\tau$, can thus be interpreted in terms of the $\mathrm{AO}$ servo bandwidth. For an $\mathrm{AO}$ system which perfectly compensates all temporal fluctuations with frequencies below $f_{c}$, but doesn't respond to higher frequencies (i.e. a bandpass filter with a sharp cut-off at frequency $\left.f_{c}\right)$, the response is equivalent to using a delay time of $1 /\left(2^{\prime}, 13 f_{c}\right)$. For a more realistic RC filter, with $3 \mathrm{~dB}$ bandwidth of $\mathrm{f}_{3 \mathrm{~dB}}$ (i.e. a bandpass filter $\left.\left(\mathrm{f} / \mathrm{f}_{3 \mathrm{~dB}}\right)^{2} /\left[1+\left(\mathrm{f} / \mathrm{f}_{3 \mathrm{~dB}}\right)^{2}\right]\right)$, the response is equivalent to using a delay time of $2.69 /\left(20.13 \mathrm{f}_{3 \mathrm{~dB}}\right)$.

4. Large Anerture. Finite Light Speed. Infinite_Bandwidth Case. In the limit of large aperture and zero delay time (i.e. infinite AO servo bandwidth), the phase variance including the finite speed of light is given by

$$
\begin{aligned}
\lim _{R \rightarrow \infty} \sigma_{\phi}^{2}=2.91 k_{0}^{2} & \stackrel{L}{d} d z C_{n}^{2}(z) w^{5 / 3}(2 z / c)^{5 / 3} \\
= & (20.132 L / c)^{5 / 3} f_{s}^{5 / 3}
\end{aligned}
$$

where a new characteristic frequency, $\mathrm{f}_{\mathrm{s}}$, is defined by

$$
f_{s}=\left[0.01956 \mathrm{k}_{0}^{2} \int_{0}^{\mathrm{L}} \mathrm{dz} \mathrm{C}_{n}^{2}(z)(w z / L)^{3 / 3}\right]^{3 / 5}
$$

As an example, for the Hufnagel-Valley $5 / 7$ turbulencu profile, using a light wavelength of $2.314 \mu \mathrm{m}$, propagating from an altitude of $41 \mathrm{kft}$ to an aimpoint located at a cross-range of $\Delta 00 \mathrm{~km}$ and an altitude of $25 \mathrm{~km}$, with the effective wind speed given by $(200 \mathrm{~m} / \mathrm{s})(\mathrm{L}-\mathrm{z}) / \mathrm{L}$, this frequency is found to be $17.96 \mathrm{~Hz}$. This gives a phase variance (including piston) of 
$0.941 \mathrm{rad}^{2}$, corresponding to a Strehl of 0.39 . This phase variance scales with wavelength to the minus six fifths power.

5. Large Aperture, Finite Light Sneed. Finite Bandwidth Case. For the large aperture limit (or when the piston component is not removed) for a given turbulence profile, a normalized phase variance can be expressed as a function of a single parameter. The phase variance is normalized to the infinite bandwidth result

$$
\lim _{R \rightarrow \infty} \frac{\sigma_{\phi}^{2}}{\left(2 n .13 f_{s} 2 L / c\right)^{5 / 3}} \frac{\int_{0}^{L} \frac{d z}{L} C_{n}^{2}(z) w^{5 / 3}\left[|\tau c / 2 L-z / L| 5 / 3-(\tau c / 2 L-z / L)^{5 / 3}\right]}{2 \int_{0}^{L} \frac{d z}{L} C_{n}^{2}(z) w^{5 / 3}(z / L)^{5 / 3}}
$$

To proceed any further, the turbulence profile, $C_{n}^{2}(z)$, must be specified. Three cases have been treated: a uniform profile, a delta function at an arbitrary position along the path, and a fairly well behaved two parameter profile. The wind speed profile will be taken as $w=v(L-z) / L$. In addition, the following variables give, a more compact notation:

$y=\lim _{R \rightarrow \infty} \frac{\sigma_{\phi}^{2}}{\left(20.13 f_{s} 2 L / c\right)^{5 / 3}}=$ normalized phase variance

$x \equiv \frac{\tau f_{o} c}{2 L f_{s}}=$ normalized delay time

With the uniform profile, $C_{n}^{2}(z)$ is constant along the path and can be taken outside the $z$ integral. In this case, $f_{d} / f_{3}=3.115$, and the normalized phase variance can be written 


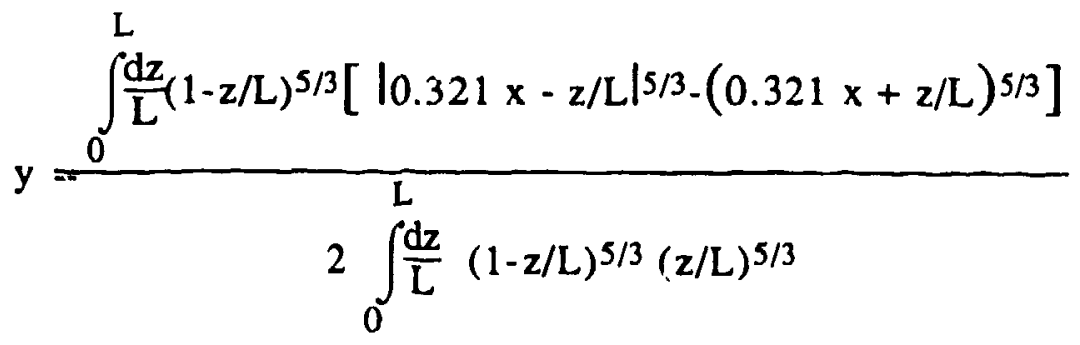

The normalized phase variance obtained by integration of Eq[14], is shown in Fig.1, expressed as a function of the single parameter, $x=\tau f_{o} c /\left(2 L f_{s}\right)$. The uniform profile gives the middle line in Fig.1.

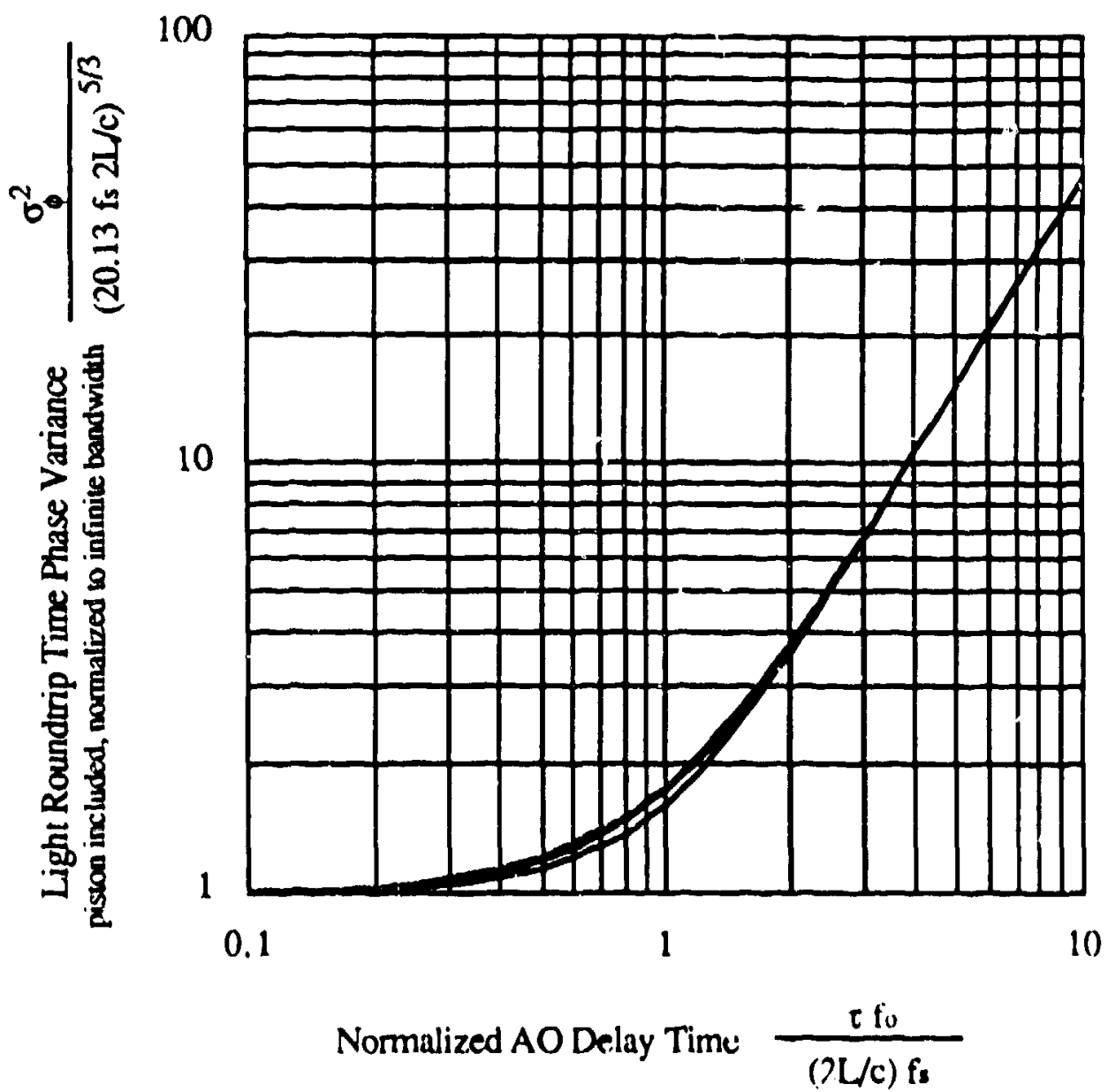

Figure 1. The normalized phase variance (including piston) including the finite speed of light and the $A($ ) bandwidth. The phase variance is normalized tu the infinite bandwidth phase variance. The three calsc:s shown are in ascending order, the delus function aurbulence profile, a uniform turbulence profile, and a rurbulence profile localized near the aperture $(p=50)$. The $\mathrm{AO}$ bandwidth is represented by a normalized delay time, depending on Greenwood's frequency, another characteristic frequency defined in the text, and the light round trip time. 
Another profile which leads to simplifications in the integrals, is that of a delta functior, i.e. $C_{n}^{2}(z)=\delta\left(z-z_{0}\right) C_{n}^{2}$. In this case, $\left(f_{o} / f_{s}\right)=L / z_{0}$, and the normalized phase variance is given by

$$
y=\frac{1}{2}\left[|x-1| 5 / 3-(x+1)^{5 / 3}\right]
$$

This normalizer phase variance is shown as the lower line of Fig.1, again as a function of the parameter $\mathrm{x}=\tau \mathrm{f}_{\mathrm{o}} \mathrm{c} /\left(2 \mathrm{Lf}_{\mathrm{s}}\right)$.

A third turbulence profile has been constructed which allows a large class of profiles to be simulated. The profile is given by

$$
C_{n}^{2}(z)=(1-z / L)^{p}(z / L)^{q} C_{n}^{2} / B(1+p, 1+q)
$$

where $p$ and $q$ are adjustable parameters which determine the profile, and $B(m, n)=\Gamma(m) \Gamma(n) / \Gamma(m+n)$ is the Beta function. For $p=q=0$, this formulation gives a uniform profile. When $\mathrm{p}$ or $\mathrm{q}$ are large, this formulation gives a delta function located at $z=L q /(p+q)$ When the turbulence is localized near the aperture, with something like an exponential fall-off with distance, the profile can be simulated by setting $q=0$, and $p=L /(e-$ folding length of the actual profile). For zenith propagation from the ground, $p$ will typically have values of 200 to 4000 . For near horizontal propagation from an airplane to a target at 25 to $50 \mathrm{~km}$ altitude, $p$ will have values of 2 to 50 . For the case of $q=0$, $\left(f_{J} / f_{s}\right)=[(p+8 / 3) B(8 / 3, p+8 / 3)]^{3 / 5}$. The normalized phase variance is then

$$
y=\frac{\int_{0}^{L} \frac{d z}{L}(1-z / L)^{p+5 / 3}\left[\left|\left(\left(p+\frac{8}{3}\right) B\left(\frac{8}{3}, p+\frac{8}{3}\right)\right]^{3 / 5} x-z / L\right| s / 3-\left(\left[\left(p+\frac{8}{3}\right) B\left(\frac{8}{3}, p+\frac{8}{3}\right)\right]^{3 / 5} x+z / L\right) s / 3\right]}{2 B\left(\frac{8}{3}, p+\frac{8}{3}\right)}[17]
$$

The normalized phase variance obtained by numerical integration of $\mathrm{Eq}[171$, is shown in Fig. 1, again expressed as a function of the single parameter, $x=\tau f_{0} c /\left(2 L f_{3}\right)$, for the case of $p=50$. This profile gives the upper line in Fig.1. There is little difference between this case and the uniform profile case. 
6. Finite Aperture. Finite Light Speed. Infinite Bandwidth Case.

When the aperture size is finite, the $J_{1}$ term of $\left.E_{c}{ }^{r} 5\right]$ must be retained. The case of sufficiently large bandwidth will be treated, so that $\tau=0$. With a delta function turbulence. profile, the normalized phase variance with piston removed will be

$$
\begin{aligned}
y & =0.8942 \int \frac{\mathrm{d} \mu}{\mu^{8 / 3}}\left\{1-\left[\frac{2 \mathrm{~J}_{1}(\mu \xi)}{\mu \xi}\right]^{2}\right\}\left[1-\mathrm{J}_{0}(\mu)\right] \\
& =\mathbf{K}(\xi)
\end{aligned}
$$

where $\xi \equiv \frac{1.59 \mathrm{D} \mathrm{c}}{20.13 \mathrm{f}_{\mathrm{s}} \mathrm{r}_{0} \mathrm{LL}}$ is a normalized aperture, and $\mathrm{r}_{0}$ is Fried's atmospheric coherence length for the path from 0 to $\mathrm{L}$, defined by

$\mathrm{r}_{0} \equiv\left[\frac{2.91}{6.88} \mathrm{k}_{\mathrm{o}}^{2} \int_{0}^{\mathrm{L}} \mathrm{dz} \mathrm{C}_{\mathrm{n}}^{2}(\mathrm{z})(1-\mathrm{z} / \mathrm{L})^{5 / 3}\right]^{-3 / 5}$

The normalized phase variance obtained by numerical integration of Eq[18], is shown in Fig.2, expressed as a function of the normalized aperture.

In the limit of small normalized aperture, the phase variance approaches the asymptotic value of

$\sigma_{\phi}^{2}=2.066\left(\mathrm{D} / \mathrm{r}_{0}\right)^{5 / 3}$ for small $\frac{1.59 \mathrm{D} \mathrm{c}}{20.13 \mathrm{f}_{\mathrm{s}} \mathrm{r}_{0} 2 \mathrm{~L}}$

This phase variance is exactly twice the phase variance of uncompensated turbilence. When the normalized aperture is sufficiently small, the phase error sensed by the beacon is uncorrelated to the required phase correction of the outgoing beam, because the turbulence changes too much during the light round trip time. The total variance is then the sum of the beacon and outgoing beam variances, i.e. twice the uncompensated result. When $\xi=0.316$, the compensated beam has the same phase variance as would be obtained with no compensation. 


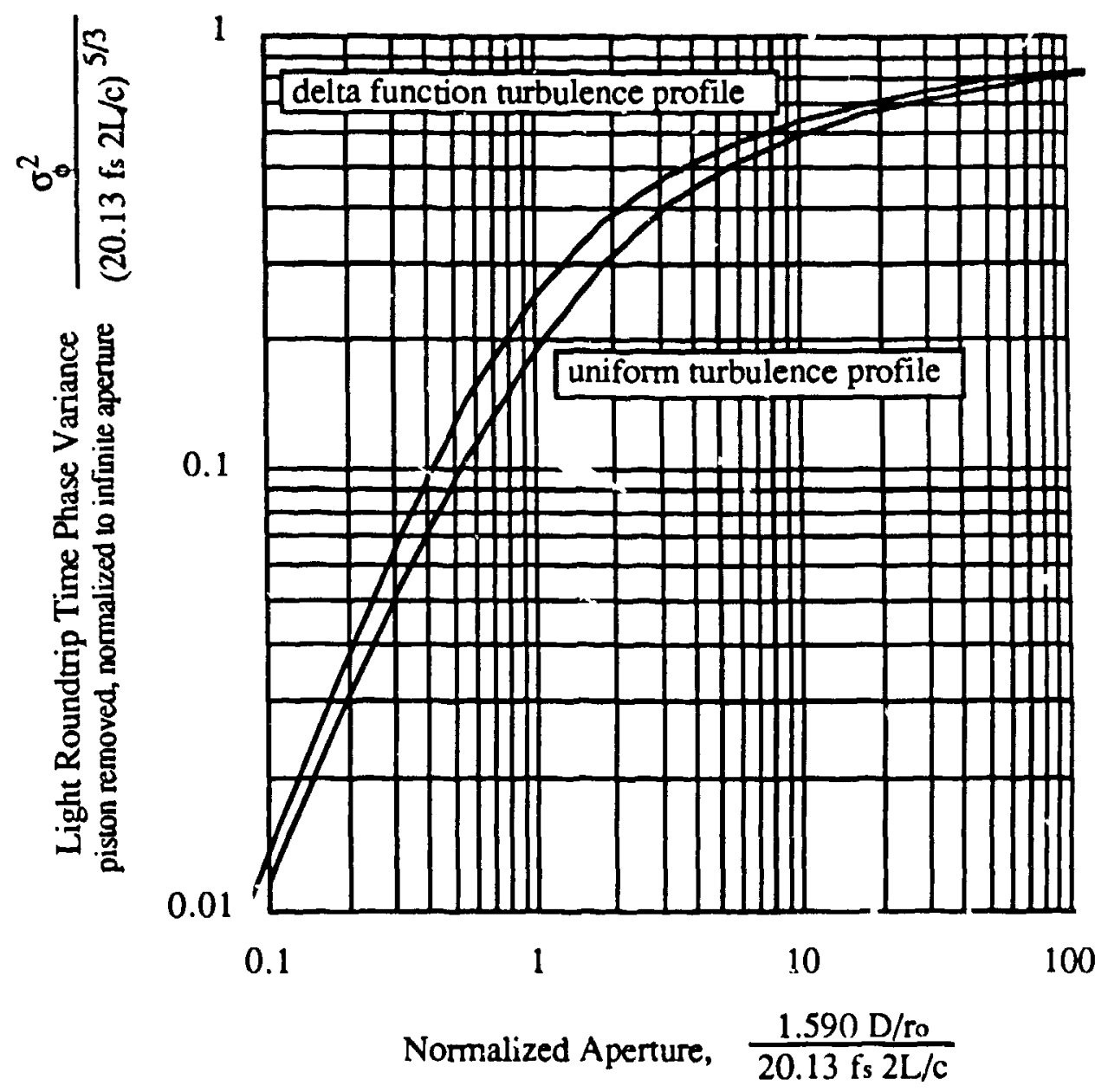

Figure 2. The normalized light round trip time phase variance (piston removed) as a function of a normalized aperture. The piston removed phase variance is normalized to the piston included phase variance, i.e. the large aperture phase variance. The aperture is normalized to the atmospheric coherence length times the characteristic frequency defined in the text times the light round trip time.

For the general profile of $\mathrm{Eq}[16]$, with $\mathrm{q}=0$, the normalized phase variance is given by

$y=\frac{1}{B\left(\frac{8}{3}, p+\frac{8}{3}\right)} \int_{0}^{L} d \gamma(1-\gamma)^{p+5 / 3} \gamma^{5 / 3} K\left(\frac{\xi}{\gamma}\left[\left(p+\frac{8}{3}\right) B\left(\frac{8}{3}, p+\frac{8}{3}\right)\right]^{3 / 5}\right)$

For $p=0$, this gives the uniform tu bulence profile result. A numerical evaluation of the normalized phase variance is also shown in Fig. 2 for this case. For values of $p$ from zero to 50, representative of propagation from an airplane along near horizontal paths, the 
resulting normalized phase variances differ from the uniform profile case by at most a few percent, and have the same asymptotes.

When $\mathrm{D} / \mathrm{r}_{0}$ is specified, the phase variance becomes a function of the single parameter $20.13 \mathrm{fs} 2 \mathrm{~L} / \mathrm{c}$. In the exarriple given befcre (Hufnagel-Valley $5 / 7$ turbulence profile, wavelength of $2.314 \mu \mathrm{m}$, propagating from an altitude of $41 \mathrm{kft}$ to an aimpoint located at a cross-range of $400 \mathrm{~km}$ and an altitude of $25 \mathrm{~km}$, plane speed of $200 \mathrm{~m} / \mathrm{s}$ ) the coherence length is $38.2 \mathrm{~cm}$. For a 2 meter aperture, this gives $\mathrm{D} / \mathrm{r}_{0}=5.21$, and the normalized phase variarce (for the $p=5$ profile) is 0.56 . Since the large aperture limit phase variance was $0.941 \mathrm{rad}^{2}$, the phase variance with piston removed is then $0.527 \mathrm{rad}^{2}$. The Strehi is then 0.59 instead of 0.39 . Fig. 3 shows the Strehl as a function of $20.13 \mathrm{f}_{\mathrm{s}} 2 \mathrm{~L} / \mathrm{c}$, for several values of $\mathrm{D} / \mathrm{r}_{0}$ using a delta function profile formulation.

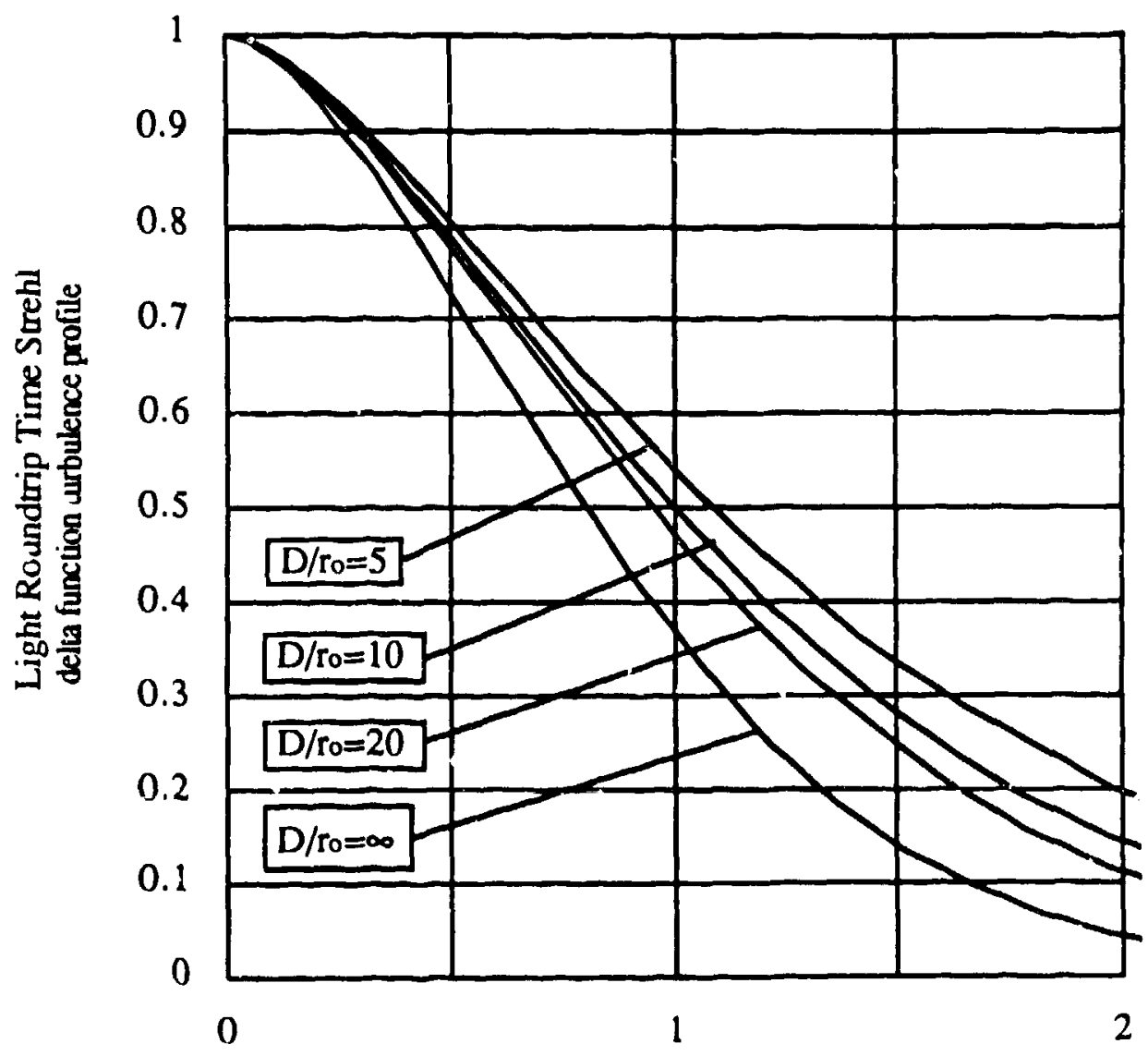

Normalized Light Round Trip Time, 20.13 fs $2 \mathrm{~L} / \mathrm{c}$

Figure 3. Light round trip Strehl as a function of a nurmalized light round trip time, for several ratios of aperture to atmospheric conerence length. The case shown is for the delta function class of turbulence profiles. The light round trip time is normalized with the characteristic frequency defined in the text. 
7. Summary. The anisoplanatic effect due to the finite speed of light, has been analyzed. It has been found that when the light round trip time is significant relative to a characteristic time, i.e. $1 /\left(20.13 f_{s}\right)$, the intensity of the beam at the aimpoint will be degraded from diffraction limited values. The beam degradation due to finite light speed w: s obtained for three cases: infinite bandwidth and large aperture; finite bandwidth and large aperture; and infinite bandwidth and finite aperture. Several turbulence strength profiles were seated, and a formulation was developed which is relatively insensitive to the details of the profile. For an airborne laser propagating on near horizonial paths, the light round trip time anisoplanatic beam degradation becomes significant at 250 to $400 \mathrm{~km}$ range, depending on turbulence strength, wavelength, and plane speed. 